\title{
Thinking outside the duct
}

Chylothorax can be an extremely challenging complication with which to deal after thoracic surgery. As many are aware, if chylothorax is allowed to progress without recognition and intervention, it can lead to malnutrition, dehydration, and immunosuppression. Although clinically significant leaks are possible after both lung and esophageal resections, they are more often found after the latter, because of the proximity of the operative field along the duct. Some traditional protocols call for an attempt at conservative management initially, before moving to a more aggressive intervention. More recently, interventional radiology-guided embolization of the thoracic duct has earned attention. The success rate of this intervention has been reported to be approximately $70 \%$ in experienced hands. ${ }^{1}$ For the remaining $30 \%$ of cases, in which duct embolization fails because of the inability to cannulate the duct, the insensitivity of the lymphoscintigraphy finding the leak, or the sometimes inexplicable failure of an apparently successful intervention, surgical therapy remains the best option.

First described by Bartolet in 1633, chylothorax had no definitive surgical treatment until Lampson ${ }^{2}$ reported a transpleural duct ligation in 1948. Until then, the mortality from chylothorax was estimated to be between $50 \%$ to $100 \%$. $^{3}$ Other early treatments included repeated aspirations of chylous pleural effusions with attempts to reconstitute the patient with chyle either orally, rectally, or intravenously, all of which were unsuccessful. Initial enthusiasm for early surgical intervention stirred by Lampson's report ${ }^{2}$ was curbed by the realization that a significant proportion of patients would not need operative intervention and therefore were unnecessarily exposed to the significant risks of reoperative morbidity and mortality. 6,7 During the next 30 years, the predominant academic discussion for the treatment of chylothorax involved identification of the optimal timing of surgical intervention, and controversies regarding the duration of conservative management and the ideal surgical technique for exposure and duct ligation were highlighted. ${ }^{5}$ Proponents of early intervention cited the advantages of prevention of malnutrition, decreased risk of mortality, and

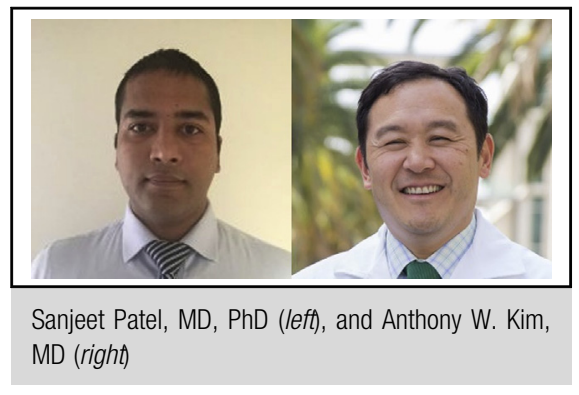

Central Message

Laparoscopic ligation of the cisterna chyli in patients who have undergone thoracic duct ligation can be an effective alternative approach to controlling refractory chylothorax.

See Article page 815

faster recovery, whereas proponents of a period of conservative management argued that, given enough time, most patients would have resolution of chylothorax without an intervention. ${ }^{6,7}$ During the period before maturation of minimally invasive thoracic surgery, open duct ligation was thought to be better than thoracoscopic clipping. ${ }^{8}$ Although the passage of time and the accumulation of experience have mitigated many of these contentious areas, the fact remains that a not insignificant proportion of patients will require additional interventions despite a duct ligation. This subset will continue to be a vexing cohort for whom innovative approaches are welcomed.

In this issue of the Journal, Diaz-Gutierrez and colleagues ${ }^{9}$ describe a minimally invasive, laparoscopic transabdominal approach for ligating the cisterna chyli that they used in 3 patients who had persistent chylothoraces after traditional interventions. They approached the cisterna chyli by exposing the lateral aorta at the celiac trunk, dissecting the inferior vena cava away from the right crus and bringing up the cisterna chyli from behind. Of the 3 patients in the series, 2 could be successfully treated with this technique. Despite the few patients described within the article, Diaz-Gutierrez and colleagues ${ }^{9}$ are to be commended for their innovative approach to this daunting problem. The passage of more time and the accumulation of additional experience will provide further evidence supporting their methods. In addition, simply raising the awareness of this approach may actually stimulate the minds of other thoracic surgeons to consider its role in their current algorithms, which currently often involve a reoperative thoracic approach. If the procedure described by Diaz-Gutierrez and 
colleagues ${ }^{9}$ is validated across other institutions, when to incorporate an abdominal approach and by what means are expected to become topics of further debate. In the meantime, Diaz-Gutierrez and colleagues ${ }^{9}$ are to be lauded for expanding the armamentarium of thoracic surgeons who have the unenviable task of treating patients with recalcitrant chylothoraces. Consistent with the ingenuity of thoracic surgeons worldwide, Diaz-Gutierrez and colleagues ${ }^{9}$ have demonstrated the importance of approaching a perplexing issue by thinking outside the duct!

\section{References}

1. Itkin M, Kucharczuk JC, Kwak A, Trerotola SO, Kaiser LR. Nonoperative thoracic duct embolization for traumatic thoracic duct leak: experience in 109 patients. $J$ Thorac Cardiovasc Surg. 2010;139:584-9; discussion 589-590.
2. Lampson R. Traumatic chylothorax; a review of the literature and report of a case treated by mediastinal ligation of the thoracic duct. J Thorac Surg. 1949;17: 778-91.

3. Shackelford RT, Fisher AM. Traumatic chylothorax. South Med J. 1938;31: 766-75.

4. Bauersfeld EH. Traumatic chylothorax from ruptured thoracic duct treated by intravenous injection of the aspirated chyle. JAMA. 1937;109:16-8.

5. Bessone LN, Ferguson TB, Burford TH. Chylothorax. Ann Thorac Surg. 1971;12: 527-50.

6. Meade RH Jr, Head JR, Moen CW. The management of chylothorax. J Thorac Surg. 1950;19:709-23.

7. Maloney JV Jr, Spencer FC. The nonoperative treatment of traumatic chylothorax. Surgery. 1956;40:121-8.

8. Kent RB III, Pinson TW. Thoracoscopic ligation of the thoracic duct. Surg Endosc 1993; 7:52-3.

9. Diaz-Gutierrez I, Rao MV, Andrade RS. Laparoscopic ligation of cisterna chyli for refractory chylothorax: a case series and review of the literature. J Thorac Cardiovasc Surg. 2018;155:815-9. 